\section{EL VÍNCULO ENTRE INNOVACIÓN MILITAR Y CIVIL: HACIA UN NUEVO MARCO DE RELACIÓN}

\author{
Jordi Molas Gallart \\ INGENIO (CSIC-UPV)
}

\section{THE LINK BETWEEN CIVIL AND MILITARY INNOVATION; TOWARDS A NEW FRAMEWORK}

\begin{abstract}
The military innovation system that emerged in the West during the Cold War, is now subject to a process of change that will fundamentally alter its relationship with its environment. The article discusses the main characteristics of this "closed" system, and analyzes the factors, both technological and strategic, that are leading to its transformation. The transition towards a more "open" system, in which the field of "defence and security policy" is no longer clearly bounded, will be long and complex, but will have important implications for the overall mode of knowledge generation and diffusion.
\end{abstract}

KEY WORDS: Innovation systems; military innovation; defence and security policy; defence industries.

\section{INTRODUCCIÓN}

El cambio tecnológico y la innovación en las industrias relacionadas con la defensa no se producen en aislamiento. La capacidad de desarrollar nuevas tecnologías para uso militar depende de la capacidad tecnológica de la economía en su conjunto; reciprocamente, muchas tecnologías desarrolladas inicialmente para ser aplicadas en el campo militar han sido después adaptadas para su uso civil. Esta relación entre innovación militar y civil es, sin embargo, cambiante y compleja. El sistema de innovación militar que se desarrolló después de la Guerra Fría tendió a cerrarse sobre sí mismo, definiendo un sistema de innovación protagonizado por un número de actores relativamente pequeño y definido (ministerios de defensa, laboratorios militares de titularidad pública, industrias de defensa...), los cuales desarrollaron sus propias dinámicas de generación y selección de nuevas tecnologías. Los procesos de selección se implementaban a través de complejos procedimientos de compra y estrictos estándares y especificaciones militares
RESUMEN: El sistema de innovación militar que se configuró durante la Guerra Fría en los países occidentales se está viendo sujeto a un proceso de cambio que va a afectar de forma fundamental la relación con su entorno. El artículo presenta las principales caracteristicas de este sistema que define como "cerrado", y analiza las razones, tanto tecnológicas como estratégicas, que impulsan un cambio de modelo. El proceso de transición hacia un sistema más abierto, en el que no existen fronteras claras que delimiten el ámbito de lo que se está empezando a definir como "política de defensa y seguridad" será largo y complejo, y afectará al modo de generación y transmisión de conocimiento en su conjunto.

PALABRAS CLAVE: Sistemas de innovación; innovación militar; políticas de defensa y seguridad; industrias de defensa.

que aseguraban el control del cliente militar sobre la configuración de los productos y sistemas que adquiría. Como respuesta a las condiciones únicas del mercado militar los mayores proveedores de sistemas militares tendieron a especializarse separando las actividades militares del resto e incluso vendiendo sus divisiones civiles a otras empresas.

Esta separación entre sistemas de innovación militar y civil está sujeta a procesos de cambio. Por ejemplo, podemos identificar multitudes de ejemplos históricos que reflejan la fluidez con la que las capacidades tecnológicas de individuos $u$ organizaciones se aplicaban indistintamente tanto a trabajos civiles como militares. Bien conocido es el caso de Leonardo da Vinci con sus diseños de armas (cañones, arcabuces, catapultas...), artículos militares (escaleras para escalar murallas, puentes para cruzar fosos...), y trabajos que hoy en día se calificarían como investigación militar (cálculos sobre la penetración de proyectiles...), que acompañaron sus múltiples trabajos artísticos y técnicos. Nicolo Tartaglia aplicó su abundante trabajo en el campo 
de las matemáticas al cálculo de trayectorias balísticas para mejorar la precisión de la artillería, y este trabajo jugo un papel muy importante en las investigaciones de Galileo y el establecimiento de las leyes de caída de los cuerpos. Estos casos son ejemplos de una práctica generalizada entre inventores, artesanos y pensadores del período preindustrial a trabajar en todo tipo de proyectos fuesen éstos militares o civiles. Las innovaciones en armamento eran a menudo desarrolladas por individuos que no formaban parte de organizaciones militares (0'Connell, 1989), y existía por tanto una gran flexibilidad en la distribución de tiempo y recursos entre tareas militares y civiles.

Con la llegada de la Revolución Industrial se establecieron relaciones estrechas a nivel institucional entre organizaciones militares y civiles. Ello facilitó que las tecnologías sociales que apoyaron los sistemas de organización jerárquicos y centralizados que caracterizaron el sistema de producción industrial fuesen frecuentemente transferidas desde el mundo militar. Así por ejemplo los ingenieros que dirigieron la construcción y gestión de la red de transporte ferroviario en los Estados Unidos eran oficiales del cuerpo militar de ingenieros (O'Connell Jr., 1985); y las formas de organización de la producción que a partir de 1815 desarrolló el US Army Ordenance Department para la producción de armas de fuego se difundieron al resto de la economía y constituyeron la base del "sistema americano de manufactura" (Smith, 1987).

La aplicación de las mismas tecnologias para fines militares y civiles (lo que hoy en día se conoce como "dualidad") no se interpretaba como problemática. Con el avance tecnológico en las sociedades industriales modernas, se intensificó el esfuerzo realizado en actividades de investigación y desarrollo tecnológico orientadas directamente al apoyo de la producción armamentista. El resultado fue un cambio en la naturaleza del conflicto armado y la destrucción de la Primera Guerra Mundial, el primer conflicto de gran escala donde se utilizaron tecnologías armamentistas de carácter industrial. En la Segunda Guerra Mundial se realizó un esfuerzo científico sin precedentes que llevó, entre otras muchas innovaciones y programas de investigación militar, al Proyecto Manhattan y al desarrollo de la bomba nuclear. Los vencedores derivaron una clara lección de esta experiencia: los resultados del esfuerzo científico y tecnológico habian sido factores cruciales en la victoria aliada. Este artículo presenta el sistema de innovación militar que surgió durante la Guerra Fría y que fue el resultado de esta constatación. Pasa a argumentar después que éste es un sistema en estado de cambio, y resume las transformaciones que ha empezado a experimentar durante las tres últimas décadas.

\section{EL SISTEMA DE INNOVACIÓN MILITAR DURANTE la Guerra Fría}

El sistema de innovación militar que emergió durante la Guerra Fría en los Estados Unidos y Europa occidental puede calificarse como jerárquico y cerrado. Jerárquico porque el cliente militar controlaba la configuración de los productos que adquiría y poseía la capacidad tecnológica y financiera para ejercer tal control. Éste era el caso en países al frente del desarrollo tecnológico militar, particularmente en los Estados Unidos. En estos países las agencias de compra militares detallaban a través de estándares, especificaciones militares (MILSPECs) y pliegos de condiciones las características técnicas de los sistemas que iban a adquirir. Algunas de estas especificaciones se desarrollaban en colaboración, formal o informal, con los contratistas principales quienes organizaban el proceso productivo y transferian los requerimientos a subcontratistas que se encargaban de manufacturar componentes y subsistemas específicos.

Las organizaciones que formaban parte de esta cadena constituían una red con intensos vínculos de colaboración pero con relativamente pocos contactos con organizaciones civiles. Esta red estaba formada por las organizaciones gubernamentales encargadas de gestionar los procesos de compra de sistemas militares (normalmente departamentos de los ministerios de defensa), laboratorios especializados de titularidad pública que ayudaban a los ministerios a desarrollar sus funciones y realizaban investigación básica y aplicada en áreas de interés para la defensa, y una red de empresas también especializadas en suministrar a cliente militar (las cuales en muchos países también eran de titularidad pública) ${ }^{1}$. Estos agentes desarrollaron una densa red de lazos que los conectaban entre sí, una cooperación a menuda tan estrecha que levantó la preocupación de políticos y analistas. El sociólogo C. Wright Mills en su famosa obra The Power Elite (Mills, 1956) ya describía la relación entre las élites económicas, 
políticas y militares que compartían intereses y una visión del mundo que Mills definía como "metafísica militar", e intercambiaban sus puestos pasando de una estructura institucional a otra.

Pocos años más tarde, el famoso discurso del Presidente Eisenhower popularizó el concepto de "complejo militarindustrial" y alertaba del peligro asociado a su irrupción. El discurso identificaba el crecimiento y especialización de la industria de armamento y sus relaciones con las instituciones públicas:

Nuestra organización militar de hoy en día poco se asemeja a la que conocieron mis predecesores tanto en tiempo de paz, como por los que lucharon en la Segunda Guerra Mundial o en Corea.

Hasta la última de nuestras conflagraciones mundiales, los Estados Unidos no tenían una industria militar. Los productores de arados podían, con tiempo y cuando así fuese requerido, producir también espadas. Pero hoy ya no podemos correr el riesgo de improvisar urgentemente la defensa nacional, nos hemos visto obligados a crear una industria permanente de armamentos de formidables proporciones. A ella debemos añadir tres millones y medio de hombres y mujeres directamente empleados en organismos de defensa. Cada año gastamos en seguridad militar más que el ingreso neto de todas las empresas norteamericanas.

Esta conjunción entre una enorme entidad militar y una gran industria de armamento es una experiencia nueva, cuya influencia -económica, política e incluso espiritual- se siente en cada ciudad, cada estado, cada oficina del gobierno federal. Reconocemos la necesidad de este desarrollo. Sin embargo, no debemos olvidar sus graves implicaciones. Nuestro trabajo, recursos, nuestra forma de vida y la estructura de nuestra sociedad están en juego.

En el desempeño de las tareas de gobierno, debemos protegernos de la adquisición injustificada de influencia, sea buscada o no, por parte del complejo militar-industrial. La posibilidad desastrosa de un incremento de poder fuera de lugar existe y persistirá (Eisenhower, 1961, traducción propia).

El término "complejo militar-industrial" ha sido, desde entonces, usado a menudo para referirse, desde una perspectiva crítica, a la influencia que sobre la toma de decisiones en temas de defensa ejercen los intereses convergentes de los industriales del sector, de las fuerzas armadas y ministerios de defensa, y de los representantes políticos principalmente de regiones en las que existen importantes centros de producción o bases militares. Estos tres grupos constituian las líneas de un "triángulo de hierro", una metáfora que se expandió en la literatura académica para referirse a la fortaleza de los vínculos que se establecían entre estos tres grupos. Adams (1982) describía la industria de defensa como una "clase especial de negocio" por la "intimidad" con la cual la industria se relacionaba con sus clientes gubernamentales. Esta familiaridad se refuerza por la facilidad con la que miembros del triángulo intercambiaban posiciones; gestores industriales pasando a ocupar puestos en el Pentágono, militares retirados encontrando empleo en las empresas de defensa, líderes industriales pasando a la política, etc. Según Adams la fortaleza de estas relaciones inhibía el control democrático sobre el proceso de compra de armamentos y la aplicación de controles de coste adecuados.

La fortaleza de estos vínculos requiere la existencia de un grupo identificable y, por tanto, relativamente estable. Los agentes que conforman el sistema de innovación militar establecian densas redes de relaciones entre ellos, y sólo vínculos más tenues con su contexto económico y social. Pero no es sólo por su composición social que el sistema de innovación militar que se desarrolló durante la Guerra Fría puede calificarse como cerrado: la organización de la investigación para la defensa ayudó a separar el mundo de la investigación e innovación militar del resto de actividades científicas y tecnológicas.

\section{La organización de la investigación militar}

El enorme esfuerzo de investigación financiado por los ministerios de defensa y otros organismos militares que se mantuvo e incrementó durante el período de la Guerra Fría $^{2}$ se gestionó a través de los mismos ministerios, fuerzas armadas y agencias afines. En algunos casos, como por ejemplo los laboratorios que heredaron la estructura del Manhattan Project y que continuaron la investigación sobre armas nucleares en los Estados Unidos, fueron y continúan siendo financiados por el Departamento de Energía, y mantienen un cierto nivel de independencia al ser gestionados por entes independientes (empresas y universidades) ${ }^{3}$. No obstante, estas situaciones eran, hasta hace poco, excepcionales. La mayoría de centros de investigación militar en los países occidentales eran de titula- 
ridad pública, gestionados directamente por organismos públicos y dotados de personal funcionario.

Fuese cual fuese la estructura institucional de los laboratorios militares, estos establecimientos se caracterizaban por formas de operar que los separaban del mundo de la investigación civil. Existían buenas razones para ello: la sensibilidad de las tecnologías que desarrollaban (explosivos, armamentos, contramedidas electrónicas...) originó un sistema de procedimientos complejos destinados a evitar la difusión de la información que estos centros generaban. El secretismo propio de las actividades de investigación militar, incluso cuando se trataba de investigación fundamental o aplicada lejos aún del desarrollo específico de sistemas militares, se constituyó en una característica diferenciadora de la I+D militar. Incluso la investigación que los ministerios de defensa y organismos afines financiaban en las universidades se realizaba bajo condiciones específicas, que, en opinión de algunos analistas, limitan la libertad intelectual y han originado un complejo "militarindustrial-académico" (Leslie, 1993).

\section{El sistema de compras públicas}

El cliente militar no sólo controlaba una cantidad de recursos muy importante para apoyar la investigación, el desarrollo y la adquisición de nuevos y complejos sistemas de armamento, sino que además estableció procedimientos de compra y de gestión y un entramado de estándares y especificaciones militares con los que controlaba los procesos de producción y las características técnicas de los sistemas que adquiría. El comprador militar se implicaba directamente en el desarrollo, diseño, e incluso producción (a través de empresas o arsenales de titularidad pública) de los sistemas que usaba. Las fuerzas armadas se encargaban también del mantenimiento de los sistemas que operaban, bien a través de centros de mantenimiento que gestionaban directamente, o a través de agencias de titularidad pública.

Así pues, en los países que lideran el ranking de gasto en compras de armamento, los clientes militares determinaban las prestaciones de los sistemas a adquirir, financiaban su desarrollo al completo, y controlaban sus detalles técnicos. Como resultado disfrutaban de la propiedad intelectual (o de derechos de uso sobre la misma) de los sistemas de armamento y de la mayoría de sus componentes y subsistemas. Los estándares militares especificaban también detalles de los procesos de producción, sistemas de control de calidad, y procesos de mantenimiento y apoyo. A este sistema los organismos responsables del proceso de compras añadieron reglas y prácticas de contratación especiales y la imposición de procedimientos únicos de inspección y control sobre los proveedores militares. Para poder jugar este papel determinante, los organismos públicos responsables de los procesos de adquisición de armamento eran apoyados por centros de investigación especializados, también de titularidad pública, con los que cooperaban estrechamente.

Por su parte, la red de proveedores que se desarrolló para responder a estas demandas tendió a especializarse, y se dotó de dilatadas estructuras administrativas para abordar los procedimientos que le venían impuestos por el cliente. Los costes administrativos impuestos por el peculiar sistema de regulaciones y procedimientos militares eran mucho más altos que los que soportaban la mayoría de las empresas operando en el resto de la economí; Melman, por ejemplo, calculó a principios de los años setenta, que en los Estados Unidos los costes de carácter administrativo incorporados al precio de un producto militar oscilaban entre el doble y el triple de los que se aplicaban a productos civiles de valor equivalente (Melman, 1974).

\section{Las características de la industria}

La industria especializada en el mercado de defensa pudo beneficiarse de los grandes programas de investigación, desarrollo y compra financiados por los ministerios de defensa. En particular, la financiación pública de la I+D le eximió del riesgo que conlleva tener que invertir fondos propios en proyectos de I+D que, por su propia naturaleza, son de resultado incierto. La industria pasó a depender de los fondos públicos para llevar a cabo sus proyectos innovadores. Ello, sin embargo, no eliminaba todos los riesgos del mercado; al contrario, la creciente complejidad y coste de los sistemas de armamento redujo progresivamente el número de proyectos y de unidades en fabricación. El número decreciente de programas, y su envergadura cada vez mayor llevaron lógicamente a la concentración industrial, al menos por lo que se refiere al número de contratistas principales. Algunas empresas se salieron del mercado y otras fueron absorbidas o se unieron, surgiendo empresas líderes que normalmente no se enfrentaban a competido- 
res nacionales. Estos sobrevivientes adquirieron una gran importancia económica y estratégica y, por tanto, fuerza e influencia política.

La proximidad de estos grupos empresariales a los centros de decisión pública reforzó los vínculos que les unían. La proximidad entre empresarios privados e instituciones públicas difuminó las fronteras entre ambas esferas. Los análisis académicos publicados entre los años sesenta y ochenta coincidian en esbozar una situación en el que el comportamiento empresarial no respondía al que cabía esperar de empresas operando en un mercado libre. Galbraith veía a las empresas militares como una extensión del departamento de defensa y viceversa (Galbraith, 1985, pp. 324-325). Melman argumentó que las empresas militares buscaban maximizar los subsidios que recibían del Estado y, por tanto, tendian a maximizar el coste de sus actividades (Melman, 1974).

Aunque Melman llevó el argumento a su extremo, la observación de que la industria militar se diferenciaba de la civil por la poca importancia que la primera otorgaba a los costes de producción, se convirtió en una de los argumentos más frecuentes en los trabajos de analistas tanto críticos como afines al sistema. A principios de los años sesenta, Peck y Scherer (1962) argumentaron que la industria militar se caracterizaba por su atención a las prestaciones de los producto que desarrollaban y producian y a la menor consideración que prestaban a los costes. La industria producia los sistemas requeridos por el cliente, con las especificaciones que éste imponía, y el cliente reembolsaba a la empresa sus costes más un margen de beneficio acordado con anterioridad. Estas prácticas, conocidas como "cost+fee", llevaron a una cultura de ingeniería y producción muy diferente de la que imperaba en los mercados civiles, donde el control y reducción de costes se erigían como uno de los factores fundamentales de competitividad. Peck y Scherer argumentaron además que, apoyadas por estos esquemas y la necesidad de alcanzar los niveles de prestaciones requeridas por el cliente, las empresas militares podían introducir productos antes de que se hubiesen solventado todos sus problemas técnicos. De esta manera en el mercado militar se introducian las innovaciones antes que en los mercados civiles, pero a un alto coste de producción y acarreando también problemas de fiabilidad y altos costes de mantenimiento, un tema que más tarde retomó Mary Kaldor desde una perspectiva crítica (Kaldor, 1982).
Las implicaciones de la relativamente poca importancia asignada a los costes de desarrollo, producción y mantenimiento, fueron más allá de su impacto sobre la propensión a la incorporación de innovaciones. Sin las restricciones de coste propias de la industria civil, y con el apoyo e incluso estímulo del cliente, la industria militar tendió a generar sistemas de complejidad (y coste) creciente, introduciendo nuevas prestaciones y capacidades en cada nueva generación de armamento. Esta práctica ha sido descrita como "gold plating" (laminado de oro) para referirse a lo innecesario de muchas de las capacidades y prestaciones que estos productos exhibian ${ }^{4}$, y su resultado como "tecnología barroca" (Kaldor, 1982): a medida que los sistemas incorporan nuevas funciones se nacian más caros de obtener, mantener $y$, también, difíciles de operar.

Esta tradición de análisis de la industria de armamento coincide en destacar, desde sus muy variadas ópticas y credos políticos, las diferencias que separaban las prácticas y cultura de la industria militar de las que caracterizaban a la industria civil. Mientras que la primera producía cantidades más pequeñas de sistemas cada vez más costosos y complejos, la segunda incrementaba los volúmenes de ventas a través del control de costes y la generación de productos cada vez más fáciles de operar y baratos de mantener. Las empresas que se habian desarrollado con una cultura de ingeniería y producción orientadas al mercado militar encontraban muy difícil su apertura a otros mercados de carácter civil. La historia de los intentos de diversificar industrias militares introduciéndolas en el mercado civil contiene algunos ejemplos de éxito pero los fracasos parecen ser más numerosos. Las diferencias en cultura, las estructuras administrativas, y las prácticas adquiridas a través de años de trabajo con un cliente dominante que jugaba un papel crucial en la especificación del producto se cuentan entre las causes que han hecho difícil el proceso de diversificación (Gansler, 1995). Ello no quiere decir que la diversificación fuese imposible o que la industria de defensa constituyese una especie de compartimento estanco. Aunque muchos autores se inclinan por subrayar los ejemplos de fracaso $0^{5}$, otros atribuyen estos casos, no a la imposibilidad práctica de implementar tales procesos con éxito, sino a la falta de compromiso por parte de los altos ejecutivos, la inercia burocrática y la falta de políticas públicas que apoyasen el proceso de diversificación (Feldman, 1999). La inexistencia de barreras

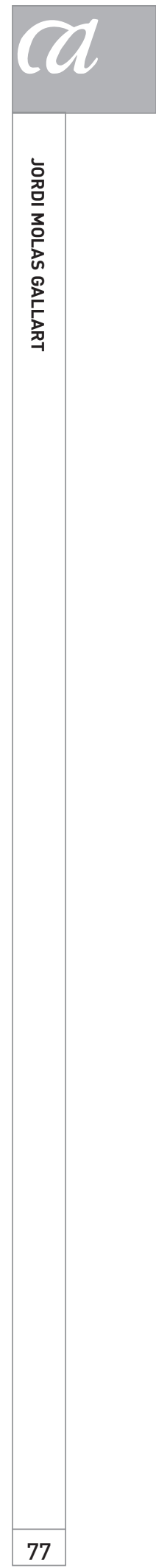

ARBOR CLXXXIV Anejo 2 [2008] 73-87 ISSN: 0210-1963 
atribuibles al uso de bases de conocimiento diferentes viene confirmada por la fluidez con la que ingenieros y científicos de "a pie" cambiaban de trabajo entre empresas militares y civiles (Lerner, 1992).

Se puede concluir por tanto, que el sistema de innovación en el área militar que caracterizó al período de la Guerra Fría en los países occidentales era dominado por un número limitado de organizaciones, la existencia de redes personales estrechas y estables conectando las "élites" que dirigían los diferentes grupos, la aplicación de reglas y procedimientos de gestión peculiares, y las diferencias que separaban las prácticas de diseño e ingeniería de la industria militar de las que caracterizaban la industria civil. Era por tanto un sistema que podía describirse de forma separada, con actores identificables y que constituían un sistema relativamente cerrado y estable. Sin embargo no se trataba de un sistema completamente cerrado: aunque las organizaciones que configuraban el sistema y las élites que las dirigían y gestionaban eran estables y fuertemente relacionadas entre sí, existía, al menos por lo que se refiere al caso norteamericano, un elemento de fluidez en las relaciones con el entorno socio-económico a través de la movilidad laboral de ingenieros y científicos.

\section{LAS RAÍCES DEL CAMBIO}

La situación que hemos definido en las secciones precedentes se puede caracterizar como la de un sistema estable. Este artículo argumenta que este sistema se encuentra actualmente en una situación de cambio; la primera pregunta que, por tanto, debemos plantearnos es cómo puede cambiar un sistema caracterizado por el dominio que sobre él ejercen pequeños grupos, o élites, que se refuerzan mutuamente. Identificaremos en esta sección cambios que se generaron en el entorno del sistema, y su relación con otras transformaciones internas al sistema. En concreto veremos cómo la modificación del entorno estratégico y de seguridad se está combinando con cambios en las relaciones entre tecnologías militares y civiles, y las respuestas políticas que esta situación genera. El resultado esta siendo la disipación paulatina de las fronteras que separaron al sistema de innovación militar de los procesos y patrones de innovación en el resto de la economía.

\section{El cambio de las relaciones entre tecnologías militares y civiles}

En los años sesenta y setenta la industria militar se encontraba al frente del desarrollo tecnológico. Las altas inversiones en I+D de defensa y la preferencia del cliente militar por conseguir prestaciones mejores llevó a las agencias militares a comprar productos en las etapas tempranas de su ciclo tecnológico, cuando debido a sus altos costes no podían ser absorbidos por los mercados civiles (De Grasse Jr., 1983, pp. 77-78). Los clientes militares jugaron un papel decisivo en el desarrollo de la industria informática (Flamm, 1987), y de muchos otros productos como los transistores, sonar, radar, sistemas de posicionamiento global, motores a reacción, maquinaria de control numérico, rodamientos, nuevos materiales, etc. ${ }^{6}$. Más tarde, al madurar las tecnologías y reducirse su coste éstas se adaptaron para su uso en mercados civiles, donde, generalmente, existía un mayor mercado potencial. Al ser los mercados civiles más amplios, la inversión en $\mathrm{I}+\mathrm{D}$ destinada a fines civiles podía crecer con rapidez y superar a la inversión militar. El ejemplo más destacado lo constituye la electrónica: si a principios de los años sesenta los únicos usuarios de circuitos integrados eran las agencias gubernamentales (que los usaban para aplicaciones en espacio y defensa), en el año 1978 el gobierno norteamericano suponía sólo un $10 \%$ del mercado doméstico, una cifra que continúo cayendo con rapidez (De Grasse Jr. 1983, pp. 84-92).

A partir de finales de los años setenta varios analistas empiezan a considerar las implicaciones de este cambio, argumentando que las tecnologías civiles estaban comenzando a aventajar, tanto en prestaciones como en coste, a tecnologías equivalentes desarrolladas con financiación militar para usos militares. Este fenómeno se detectó en varios sectores, incluyendo la electrónica, el sector aeroespacial y los nuevos materiales (Cuthbertson, 1983, p. 11). Al desplazarse las fuentes de generación de nuevas tecnologías fuera del ámbito del sistema de innovación militar, las debilidades percibidas en la industria civil pasaron a interpretarse como riesgos para la seguridad (Manufacturing Studies Board, 1987). Este argumentó se presentó con especial fuerza en los Estados Unidos y coincidió, paradójicamente, con el fuerte incremento en gasto militar (incluyendo I+D) que impulsó el gobierno del Presidente Reagan. Los analistas apuntaron que, a pesar del gran esfuerzo que se estaba invirtiendo en investigación militar, la 
fortaleza de la industria de la defensa debería descansar en una base tecnológica civil fuerte (U.S. Congress Office of Technology Assessment, 1989). Por tanto, no era suficiente (y quizás ni tan sólo necesario) incrementar los gastos en I+D militar sino que había que impulsar la base científica y tecnológica del país y establecer mecanismos para que los avances que se lograsen pudieran usarse para mejorar también la seguridad nacional. Estos mecanismos resultan, sin embargo, difíciles de establecer: significan, por ejemplo que el comprador militar debe abandonar su costumbre de controlar el desarrollo y ostentar derechos de propiedad intelectual sobre los componentes y subsistemas que integran los sistemas que adquiere y opera.

Además de los cambios en los sectores que el sector de defensa había apoyado en su origen, los últimos años del siglo XX fueron testigo de la emergencia de nuevas tecnologías industriales que, a pesar de haber sido financiadas en su mayor parte para fines civiles, tienen importantes implicaciones para el campo de la defensa y la seguridad. Por ejemplo, áreas de conocimiento tradicionalmente englobadas en el campo de la nanotecnología pueden aplicarse al desarrollo de sensores distribuidos, armas no metálicas y sistemas armados autónomos (Altman, 2006), y los avances en biotecnología pueden generar nuevos patógenos y difundir las tecnologías para su generación, almacenamiento y dispersión, un desarrollo de evidente y preocupante aplicabilidad tanto para fuerzas armadas convencionales como grupos terroristas.

Al abrirse el abanico de sectores y áreas del conocimiento relevantes para la defensa las organizaciones militares deben buscar nuevos proveedores y acceder a nuevas fuentes de conocimiento. Sin embargo, en su intento de expandir su elenco de proveedores, el cliente militar se encuentra con que los complejos procesos y detalladas regulaciones que había desarrollado para gestionar la compra de sistemas militares durante la Guerra Fría, emergen ahora como una dificultad que se opone a la expansión del número de proveedores operando en el mercado. Los potenciales nuevos proveedores de conocimiento y tecnologías tienen normalmente un ámbito de actuación ya establecido en los mercados civiles, y el cliente militar aparece tan sólo como un cliente más. En esta situación el cliente militar pierde su fuerza para imponer los peculiares sistemas de compra y gestión que representaban una piedra angular del sistema de innovación militar que se desarrolló durante la Guerra Fría. De forma parecida, para poder acceder a las tecnologías comerciales que han convertido en obsoletas a sus equivalentes militares, el cliente militar debe reducir el alcance y magnitud de las especificaciones y regulaciones propias; de lo contrario los proveedores civiles, cuyos principales mercados residen en otros ámbitos, no considerarán suficientemente atractivo al cliente militar.

Estos cambios hacen que los peculiares procesos de compra y gestión, basados en el uso extensivo de especificaciones y estándares militares, deban abandonarse. De igual modo, para incorporar nuevos conocimientos y tecnologías los organismos públicos de investigación en defensa se ven obligados a abrirse a nuevos campos de trabajo y flexibilizarse y, para ello, renunciar a una gran parte de las prácticas de aislamiento y secretismo que les caracterizaban.

\section{Seguridad y defensa: dos ámbitos convergentes}

El segundo factor que impulsa el cambio en la forma en que el sector de defensa generaba innovaciones viene de la mano de cambios en la percepción de las amenazas a la seguridad nacional y la naturaleza del conflicto armado, y de las transformaciones institucionales que tal cambio está empezando a ocasionar. Con el final de la Guerra Fría la posibilidad de un enfrentamiento militar convencional y masivo entre las fuerzas armadas de los dos bloques se disipó. En cambio, durante los años noventa las fuerzas de la OTAN y de sus países miembros empezaron a tomar parte en una serie de conflictos regionales caracterizados por la emergencia de guerras civiles, la fragmentación del poder del Estado y su "distribución" entre grupos opuestos, el paso del control regional y local a grupos armados relativamente pequeños (milicias, "señores de la guerra"), y la participación de estos grupos en una gran variedad de actividades que incluyen la guerra de guerrillas, el tráfico de armas y estupefacientes, el chantaje sistematizado, y la imposición de sus propias normas de comportamiento sobre las zonas que controlan?. En estos "nuevos" conflictos la violencia se dirige mayoritariamente hacia la propia población local, y el papel de las fuerzas armadas pasa de ser una fuerza de combate tradicional al de una organización que debe mantener la seguridad interna al mismo tiempo que se ve envuelta en un conflicto de "baja intensidad".

La segunda transformación significativa se ha dado con la aparición de lo que se ha dado en Ilamar "nuevo terroris- 
mo". El término se usa para referirse a la emergencia de grupos entre cuyos objetivos se cuentan la devastación de infraestructuras y poblaciones civiles. Los ataques del 11 de septiembre se mantienen como ejemplo de la escala de destrucción que estos grupos persiguen; éste fue el ataque más mortífero lanzado contra el continente norteamericano desde el siglo XIX y, notablemente, el resultado de una operación organizada por civiles usando aviones comerciales como arma. La determinación con la que los nuevos grupos buscan causar números elevados de víctimas civiles y la disponibilidad de un amplio surtido de tecnologías comerciales, tanto nuevas como maduras, que pueden adaptarse a tales fines, han generado preocupación sobre las formas que los terroristas pueden usar para realizar ataques masivos.

Esta situación, y en particular la magnitud de los ataques del 11 de septiembre y el reguero de otros ataques terroristas, por ejemplo, en Gran Bretaña, Indonesia y España han incrementado la percepción de una nueva e importante amenaza a la seguridad e impulsado el desarrollo de una nueva agenda política que fusiona objetivos tradicionales de defensa militar con objetivos de seguridad nacional en lo que se está empezando a llamar "política de seguridad y defensa". Este desarrollo tiene gran importancia: el tipo de sistema que caracterizó a la Guerra Fría y que hemos discutido en la primera parte de este artículo se caracterizaba por una clara distinción entre la defensa de carácter militar contra las amenazas presentadas por otras tuerzas armadas extranjeras, y la seguridad nacional de carácter interno. Diferentes organizaciones gubernamentales y diferentes ministerios se encargaban de estas dos áreas. Normalmente, los presupuestos asignados a los ministerios encargados de la seguridad doméstica y a las fuerzas policiales o paramilitares que se encargaban de estas tareas eran mucho más pequeños que los presupuestos de defensa. Como se resultado se generaron formas institucionales diferenciadas, con su propia estructura, cultura y redes de proveedores.

La separación institucional continúa entre las organizaciones que se encargan primordialmente de la defensa militar contra amenazas extranjeras y las encargadas de la seguridad doméstica; pero es ésta una separación que se está difuminando paulatinamente. En primer lugar, el concepto de "seguridad y defensa" ha hecho su entrada con fuerza en el discurso político de muchos países y organizaciones internacionales, y también en el mundo académico. En la práctica el nuevo tipo de conflictos armados al que ya nos hemos referido implica que las fuerzas militares se han visto repetidamente envueltas en operaciones en las que han terminado trabajando como fuerzas de seguridad en los países en los que operan (los casos de las guerras balcánicas y la guerra de Iraq constituyen buenos ejemplos de esta situación). Naturalmente, las fuerzas armadas también se preocupan de las amenazas extranjeras que pueden proyectarse a través de ataques terroristas sobre el territorio nacional.

Este tipo de desarrollo empieza a romper las relativamente pequeñas comunidades de actores que hemos descrito anteriormente y que constituían uno de los elementos caracterizadores del sistema de innovación cerrado de la Guerra Fría. Como veremos en la siguiente sección las agencias de seguridad está creciendo en importancia y permitiendo la entrada de nuevos actores que participan en la elaboración de políticas en un área en la que también concurren los organismos militares tradicionales, cuyas responsabilidades se están ampliando para dar cabida a nuevas tareas ligadas con el campo de la seguridad nacional.

\section{Las respuestas a los cambios y la emergencia de un nuevo sistema}

\section{El ámbito de la "seguridad y la defensa"}

El cambio en el contexto de la seguridad nacional ha llevado a la emergencia de nuevas agencias y ministerios de seguridad y a la redefinición de las tareas y responsabilidades de los ya existentes. Un ejemplo destacado de nueva organización creada a tal efecto es el Department of Homeland Security (DHS) en los Estados Unidos. Un ejemplo de redefinición de tareas de organismos ya existentes lo constituye la forma en la que la Unión Europea está adoptando responsabilidades en el campo de la seguridad. De particular interés para el tema que nos ocupa, es que estos cambios institucionales llevan asociados nuevos programas y actividades de investigación. Así por ejemplo, el presupuesto de I+D del DHS norteamericano subió rápidamente hasta alcanzar 1.300 millones de dólares en 2006, una cifra que si bien parece pequeña cuando la comparamos con el presupuesto de I+D del Pentágono (uno 72 mil millones anuales de dólares) supera al gasto anual en I+D de la mayoría de ministerios de defensa de los 
países europeos. Esta inversión permite al DHS desarrollar un programa de investigación ambicioso en el que participan tanto empresas y organizaciones ligadas al campo de la producción militar, como empresas que han operado tradicionalmente en mercados civiles.

En el ámbito europeo, las instituciones de la Unión Europea, cuyo mandato se había limitado históricamente a actividades de tipo civil, han adquirido progresivamente durante la última década responsabilidades que se enmarcan dentro de un área ambiguamente definida como de seguridad. Desde el punto de vista de actividades de investigación, el 7. Programa Marco incluye por primera vez un programa sobre "investigación en seguridad" cuyos objetivos incluyen la "mejora de la seguridad de los ciudadanos" y la "mejora de la innovación y la competitividad en las industrias relacionadas con la seguridad" (CORDIS, 2006). Resulta interesante observar que el programa no define lo que se entiende por "seguridad" ni el grupo de empresas "relacionadas" con la misma. La Comisión Europea ha aceptado que el concepto de seguridad es difícil de definir, y que se trata de un "concepto en evolución".

Por el momento, tanto el DHS norteamericano, como la Comisión Europea coinciden en delimitar un campo amplio de actividades y objetivos en el seno de lo que entienden como políticas de seguridad. El programa de investigación sobre seguridad del 7. Programa Marco va más allá de la lucha contra el terrorismo para incluir la lucha contra el crimen organizado, y el desarrollo de medidas para prevenir y abordar los desastres naturales. Igualmente el DHS aborda responsabilidades que están lejos de limitarse a la lucha antiterrorista, e incluyen la lucha contra los desastres naturales y sus efectos, el control de fronteras y la operación del servicio de aduanas, entre otros. La diversidad de actividades que se incluyen dentro del ámbito de la "seguridad" diluye la distinción entre la defensa contra actos violentos motivados políticamente (el campo tradicional de actividad de los ministerios de defensa y del interior) de la protección contra desastres naturales, y el control de fenómenos sociales como la inmigración. Existen razones para aglutinar estas actividades en un mismo departamento. El control de fronteras es en sí mismo un elemento importante de la lucha antiterrorista. Ataques terroristas contra infraestructuras o recursos naturales pueden equipararse por sus efectos a los de ciertos desastres naturales. Es por tanto lógico que exista un grado de confluencia entre las agencias y los programas destinados a atajar tan diferentes fenómenos.

Desde una perspectiva tecnológica el resultado es un gran incremento en el número de campos de investigación de interés para los organismos encargados de la seguridad y la defensa. Muchas de las tecnologías desarrolladas por organizaciones civiles para cubrir necesidades civiles son ahora consideradas importantes desde la óptica de la seguridad, bien por presentar vulnerabilidades que podrían ser aprovechadas por grupos terroristas, o capacidades que podrian desarrollarse para su uso en un contexto de lucha armada o en la lucha, por ejemplo, contra los efectos de las catástrofes naturales. Las comunidades científicas que trabajan para las agencias de seguridad y defensa se han visto consecuentemente ampliadas. Además, al ampliarse la percepción de que amplios campos de la investigación científica y tecnológica (desde la biotecnología a la ingeniería civil) tienen importantes implicaciones para la seguridad, se están intensificando los controles formales sobre los flujos de conocimiento y la transferencia de tecnologías, extendiéndose a las actividades de investigación e incluso de enseñanza.

Estos cambios ocasionan situaciones de confusión institucional. Por ejemplo, en el caso europeo, los documentos de la Comisión Europea aseguran que sus actividades de I+D en el campo de la seguridad mantendrán "exclusivamente" una orientación civil, para después reconocer que la investigación cubrirá áreas de tecnología dual (de aplicación tanto militar como civil) e incluirá cooperación con la Agencia Europea de Defensa. Por una parte la Comisión Europea intenta mantener el carácter civil del Programa Marco ante la oposición de algunos países miembros y de grupos políticos a la "militarización" de las actividades comunitarias. Este intento de mantener separadas las actividades civiles de las de defensa (con contribución militar) ha dado lugar a un complejo proceso de cambio institucional Ileno de dificultades y ambigüedades. Cuando el Tratado de Maastricht introdujo la Política Exterior y de Seguridad Común (PESC) como uno de los elementos de la Unión Europea, se asignó a la Unión Europea Occidental (UEO), y no a la Comisión la elaboración e implementación de las decisiones de la Unión Europea (UE) con implicaciones en el campo de la defensa. Este intento de incluir temas de seguridad y defensa en la agenda de la Unión Europea bajo una 
estricta separación institucional fue abandonado cuando en la reunión de Colonia en 1999, el Consejo de la UE transfirió las actividades de la UEO a la UE y nombró a Javier Solana como "Alto Representante" de la UE para la PESC. La sopa de letras que surge (y que se irá complicando con la creación en el 2004 de la Agencia Europea de la Defensa-ADE, y la elaboración de una Política de Seguridad y Defensa Europea) refleja las dificultades prácticas de separar, en el nuevo contexto internacional, las actividades de carácter militar de las de carácter civil. La falta de definición clara del concepto de "seguridad" y su relación con la "defensa" y las "actividades militares" permiten, por ejemplo, a la Comunidad Europea la financiación de actividades de $I+D$ directamente relevantes para la industria militar y al mismo tiempo mantener que el carácter del Programa Marco continúa siendo "exclusivamente civil".

La apertura y la confluencia entre diferentes tareas tiene también otras expresiones institucionales: algunos ministerios de defensa están expandiendo formalmente sus objetivos. En España, por ejemplo, la "Unidad Militar de Emergencias" creada en octubre de 2005 y operacional desde verano de 2007, está encargada de operar en todo el territorio español en casos de grave riesgo, calamidades $u$ otras necesidades públicas. La UME ha participado en la extinción de incendios forestales y es un ejemplo de la extensión de las fuerzas militares para cubrir, como una de sus misiones explícitas, el apoyo a la población civil en el caso de catástrofes naturales y otras situaciones de "grave riesgo" que quedan sin explicitar.

Nos encontramos por tanto en un contexto en el que nuevas agencias entran a operar en áreas convergentes con las que tradicionalmente se consideraban de cariz militar, y los ejércitos se ocupan formalmente de tareas de seguridad nacional y protección civil. Al disiparse la distinción entre "seguridad" y política militar, la naturaleza cerrada del sistema de innovación militar empieza a desaparecer. Empresas tradicionalmente militares participan activamente en el creciente mercado de sistemas de seguridad para nuevos o reforzados clientes públicos, las actividades de las agencias militares se abren para cubrir nuevas tareas civiles, y consecuentemente su demanda de productos y sistemas se amplia para cubrir lo que hasta el momento se consideraban actividades dominantemente civiles. Este último es uno de los factores, aunque no el más impor- tante, que impulsan a los ministerios de defensa a abrir sus procesos de compra para atraer a nuevos proveedores. Éste es el tema que abordamos seguidamente.

\section{La reforma de los procesos de compra}

Las agencias y departamentos gubernamentales encargados del desarrollo y compra de sistemas de armamento han impulsado en muchos países iniciativas de reforma de los procesos de contratación y mantenimiento. Estos procesos han recibido especial atención principalmente en los Estados Unidos y Gran Bretaña, donde han sido objeto de discusión y análisis detallado.

El principal factor que está impulsando las políticas de cambio es el reconocimiento de las posibilidades abiertas por el desarrollo de tecnologías civiles que ofrecen soluciones mejores y más económicas a muchos de los problemas técnicos que plantea el desarrollo de nuevos sistemas de defensa. Desde esta perspectiva el objetivo es desarrollar políticas que permitan y estimulen el uso de componentes y subsistemas comerciales; sin embargo, este objetivo plantea problemas mucho más serios de los que podría parecer a simple vista. No se trata simplemente de sustituir un tipo de productos por otros, o de ampliar la base de proveedores, sino de cambiar la forma en la que tradicionalmente las agencias militares han participado en el desarrollo y producción de sistemas militares.

En primer lugar, el gran número de especificaciones y estándares militares cuyo cumplimiento se requería en prácticamente todas las compras de sistemas militares deben abandonarse siempre que sea posible (Molas-GaIlart, 2001). En segundo lugar, los procedimientos contractuales deben simplificarse si se quiere atraer nuevos proveedores cuyo ámbito de actividad principal no se encuentra en los mercados militares. En tercer lugar, el uso de componentes y subsistemas de origen comercial requiere la adopción de contratos de mantenimiento a largo plazo, la adopción de estimaciones de coste que incluyan todo el ciclo de vida de un sistema, y la introducción de sistemas de "gestión de la obsolescencia"; ello se debe al riesgo adicional que comporta el uso de componentes comerciales sobre los cuales ni el consumidor ni el sistemista pueden controlar la propiedad intelectual y el detalle de sus características técnicas. 
Así por ejemplo podría darse el caso de componentes comerciales que sean más económicos en el momento de la compra, pero de fiabilidad menor, de forma que al sumar el coste de mantenimiento al de adquisición el resultado sea un coste que a la larga resulte mayor. Es por ello, que cuando se incorporan componentes comerciales es necesario introducir contratos a largo término que incluyan los costes de mantenimiento, y que al tomar decisiones sobre la compra de subsistemas y componentes éstas se realicen sobre la base de estimaciones del coste durante un período extenso de uso del sistema.

De forma parecida, muchos de los componentes comerciales que pueden introducirse en sistemas de armamento tienen ciclos de vida muy cortos; por ejemplo, nuevas generaciones de microprocesadores surgen constantemente mientras que un sistema complejo de armamento permanece en uso durante décadas. Ello significa que al introducir componentes comerciales en sistemas militares, los usuarios deben planificar las necesidades futuras de tales componentes y su estrategia de adquisición; bien a través de la compra y almacenamiento de existencias, o de la negociación de acuerdos que permitan su producción futura cuando ya se hayan retirado de los mercados comerciales. Sin embargo, a medida que el comprador militar pierde control sobre el diseño y configuración del sistema, la gestión de los derechos de propiedad intelectual durante el ciclo de vida se convierte en un tema de disputa entre proveedores y clientes. En resumen, se requiere un cambio profundo de la forma en que se adquieren sistemas de armamento y sobretodo del nivel de control que el comprador ejerce sobre el proceso. Ello requiere modificaciones, no sólo de legislación, reglamentos y prácticas, sino también de cultura.

Si este proceso de reforma tiene éxito, el número de proveedores de sistemas de defensa (y de seguridad) crecerá y el consumidor perderá el control sobre la configuración de los sistemas que opera. La incorporación de componentes y subsistemas desarrollados inicialmente para aplicaciones civiles, supone que el cliente militar pasa a ser un comprador más de empresas que ya no son especialistas en la producción de sistemas militares. Proveedores no especializados ganan importancia en el mercado de defensa, y la separación que anteriormente se podía realizar entre "industrias militares" e "industrias civiles" deja de ser posible.
La reforma de los laboratorios de defensa

Otra de las características del sistema de innovación militar durante la Guerra Fría fue el importante papel desempeñado por los centros públicos de investigación especializados en I+D militar. La creciente importancia de las tecnologías de origen civil y el descenso en los presupuestos de I+D militar durante los años noventa impulsaron su reforma. Las políticas de cambio perseguían:

1. Abrir los laboratorios militares a la comunidad científica y tecnológica civil y así incrementar su potencial para identificar tecnologías de origen civil y adaptarlas a aplicaciones militares.

2. Explotar en nuevos mercados sus capacidades científicas y tecnológicas y de este modo compensar el descenso en las inversiones gubernamentales en $\mathrm{I}+\mathrm{D}$ de defensa.

Aunque existen importantes diferencias entre paises por lo que se refiere a la estructura y gestión de los laboratorios de defensa, la tendencia a abrir sus actividades hacia nuevos campos y mercados es generalizada. En Gran Bretaña, la privatización de aproximadamente dos tercios del antiguo laboratorio de defensa ha llevado a la empresa resultante (Qineti0) a una expansión de sus actividades y la penetración de nuevos mercados. Qineti0 ha desarrollado productos para los mercados de seguridad (comercializando sistemas de seguridad aeroportuaria, sistemas de observación de espacios públicos, etcétera), ha creado empresas para comercializar en mercados civiles algunas de sus invenciones y ha intensificado sus actividades de comercialización de patentes, al mismo tiempo que mantiene su actividad principal en el campo militar.

En los Estados Unidos los laboratorios de armamento nuclear $^{8}$ adoptaron nuevas misiones al finalizar la Guerra Fría (Crease, 1993) y redujeron su dependencia de la actividad nuclear 9 . Los centros ampliaron sus objetivos, firmaron acuerdos de cooperación científica con empresas, abrieron sus laboratorios a usuarios civiles, prestaron asistencia técnica a pequeñas y medianas empresas, y crearon empresas para comercializar sus innovaciones en mercados civiles; todo ello con el propósito de diversificar sus fuentes de financiación. 
En Francia, por citar otro ejemplo, el proceso de cambio gira en torno a dos ejes. En primer lugar, la reforma de la agencia encargada del desarrollo y compra de sistemas de armamento: la Délégation Générale pour l'Armement (DGA), creada por el General De Gaulle. La DGA es la institución central en la estrategia tecnológica e industrial del ministerio de defensa, del cual depende. Participa en todos los aspectos del desarrollo y producción de nuevos sistemas de armamento, incluyendo la realización de investigación científica y estratégica a través de varios centros de investigación que la misma DGA controla. A partir de mediados de los noventa el Ministerio de Defensa francés decidió reducir el papel de la DGA, convirtiéndola de una "agencia de programas" a una "agencia de compras", con el fin de instaurar sistemas institucionales más abiertos que facilitasen la participación en proyectos conjuntos europeos y la participación de nuevas organizaciones en los procesos de investigación y desarrollo de sistemas de defensa. En segundo lugar, el papel de los laboratorios públicos en la I+D de defensa ha descendido rápidamente. Entre el 1998 y el 2001 el porcentaje del presupuesto de investigación y tecnología de defensa que absorbian dos organismos públicos de investigación (el Comissariat à I'Energie Atomique y ONERA) se redujo del $65 \%$ a un $21 \%$. Las empresas y organismos externos como universidades han cubierto el hueco, pasando de representar un $27 \%$ a un $68 \%$ de los gastos ${ }^{10}$. Estas cifras representan un cambio muy sustancial en el transcurso de sólo tres años.

Existe por tanto, en paises muy diversos y con tradiciones políticas diferentes, un grado de convergencia en el carácter de las reformas del sistema público de investigación en defensa. Aunque el éxito a largo término de estos cambios esté aún por determinar, las nuevas políticas han llevado a la apertura de una parte sustancial de las instalaciones de los laboratorios militares a la interacción con empresas civiles y a la entrada de nuevas organizaciones en las actividades de investigación financiadas con cargo a defensa.

\section{Conclusiones}

Este artículo ha resumido las transformaciones en las condiciones económicas y estratégicas que están transformando el sistema de innovación militar en los inicios del siglo XXI. Estos cambios van a ser lentos y difíciles pero profundos ya que implican la alteración de la forma en que la innovación militar y la civil se relacionan entre sí, y requieren profundas transformaciones institucionales y culturales. La participación de nuevas organizaciones en la producción de sistemas de armamento, la ampliación del ámbito de actividad de las organizaciones militares para incluir tareas ligadas a la seguridad nacional, la emergencia de nuevas organizaciones de seguridad que tienen en parte una vertiente militar, y la extensión de las tecnologias y actividades de interés para la seguridad y la defensa, se están dando de forma progresiva, pero han empezado ya a difuminar la diferenciación entre tecnologías militares y civiles, y a abrir el sistema de innovación militar a nuevos actores. Se trata de un cambio que lleva al sistema de innovación militar de una situación cerrada a un sistema abierto en el cual no existen ya líneas divisorias claras que separen a las tecnologías militares del resto. Las fuentes de innovación militar han pasado de ser internas al sistema (los requerimientos y especificaciones que emanaban del consumidor militar) a encontrarse en la industria civil, y las empresas de carácter civil están jugando cada vez un papel más relevante en la producción de sistemas de armamento y defensa.

Las instituciones que participan en este cambio van más allá de las directamente relacionadas con el mundo militar (fuerzas armadas, ministerios de defensas, agencias de compras, laboratorios de defensa, empresas especializadas...) para incluir un grupo más amplio y creciente de organizaciones (ministerios de seguridad nacional, organizaciones internacionales, empresas civiles...). Pero la respuesta institucional no ha sido automática. Muchos analistas se mantienen escépticos sobre el éxito de las iniciativas de reforma impulsadas en los Estados Unidos y mantienen que las industrias militares y el Pentágono continúan sustentando una relación análoga a la que han mantenido durante las décadas precedentes. Sin embargo, incluso iniciativas aparentemente limitadas, como la reducción en el número de especificaciones y estándares militares tienen consecuencias importantes sobre la naturaleza y estructura de la producción militar y requieren la implementación de otros cambios adicionales (como la introducción de contratos que incluyan el mantenimiento de los sistemas a largo plazo).

Aunque los principales proveedores militares continúan siendo casi los mismos de hace una década (una vez hemos 
tomado en consideración las fusiones y compras de empresas), su relación con el cliente militar está cambiando. Así, por ejemplo, al pasar el comprador una parte de la responsabilidad de financiación de la $\mathrm{I}+\mathrm{D}$ a las empresas proveedoras surgen con mayor frecuencia conflictos sobre la gestión de los derechos de propiedad intelectual (MolasGallart y Tang, 2006). Las relaciones entre los proveedores tradicionales y el cliente militar se vuelven más tensas, al mismo tiempo que surgen nuevas empresas cuya capacidad principal es la gestión e implementación de proyectos que impliquen el mantenimiento a largo plazo de sistemas e infraestructuras. Las semillas del cambio están ya plantadas, aunque sus efectos no sean aún evidentes.

La inercia institucional de las agencias y organizaciones militares se sustenta sobre un sistema de procesos y regulaciones distintivos que regían, y en parte aún rigen, el desarrollo y compra de armamentos. La confluencia de las agendas de seguridad y defensa dará paso a nuevas coaliciones entre grupos que participen en la definición e implementación de nuevas agendas políticas y creen nuevas arquitecturas institucionales. Dada la confusión presente sobre los papeles a desempeñar por los organismos públicos, sean nuevos o ya existentes, en el ámbito de la "seguridad y defensa", estas nuevas coaliciones no van a solidificarse rápidamente ni van a encontrar una plasmación institucional inmediata. Sin embargo, están ya abriendo el abanico de participantes en lo que había sido durante la Guerra Fría un ámbito de actuación política fácilmente identificable y cuyos actores podian reconocerse con cierta claridad. La otra cara de esta moneda es que las regulaciones y restricciones sobre los flujos de conocimientos y tecnología que hasta ahora se aplicaban a las tecnologías militares y a las Ilamadas "tecnologías duales", se están expandiendo a amplios campos de conocimiento. A medida que el sistema de innovación en el campo de la defensa y la seguridad se abre a nuevos actores y tecnologías, los sistemas de regulación del conocimiento científico y tecnológico se expanden para cubrir áreas que hasta hace poco se consideraban como esencialmente civiles.

\section{NOTAS}

1 El término "sistema cerrado" fue acuñado por Paul Edwards (1997) añadiendo que el carácter de los productos que el sistema generaba apoyaba la creación de sistemas de control centralizados, acompañados de un "discurso" que subrayaba las virtudes de la dirección centralizada.

2 En las décadas tras el final de la Segunda Guerra Mundial en los Estados Unidos, por ejemplo, los gastos en $I+D$ de defensa se han colocado consistentemente cerca de los 2/3 del gasto total gubernamental en I+D. En Europa la países con un gasto relativo más alto han sido Francia y Gran Bretaña, cuyo gasto en $\mathrm{I}+\mathrm{D}$ de defensa osciló alrededor del 50\% del gasto público en $\mathrm{I}+\mathrm{D}$ durante las décadas de la Guerra Fría, habiendo descendido recientemente.

3 Los laboratorios son de titularidad pública pero son operados por contratistas contratados por el Departamento de Energía. En Gran Bretaña el centro de investigación sobre armas nucleares (Atomic Weapons Establishment) se organiza de forma parecida aunque su titularidad corresponde al Ministerio de Defensa británico.

4 Véase por ejemplo el análisis de Hampson sobre el desarrollo y compra de varios sistemas de armamento norteamericanos (Hampson, 1989). Hampson menciona por ejemplo el caso del avión de combate F-16, inicialmente un avión pequeño que el Secretario de Defensa norteamericano James Schlessinger aprobó debido a su simplicidad y bajo precio. Una
Recibido: 9 de enero de 2008

Aceptado: 19 de marzo de 2008 
vez aprobado las fuerzas áreas empezaron a introducir cambios que incrementaron su complejidad y coste, incluyendo modificaciones que suponían la incorporación de dos toneladas de sistemas electrónicos.

5 Stear, por ejemplo, analiza el caso de Boeing y encuentra ejemplos de intentos fallidos de diversificación en los años veinte, setenta y noventa (Stear, 1997).

6 Existen muchas fuentes que ofrecen listas de productos que han sido inicialmente desarrollados gracias a la inversión de ministerios de defensa y agencias militares y que después se ha difundirlo al mundo civil (De Lauer, 1984; 1937).

7 Este tipo de conflicto ha sido caracterizado como una nueva forma de guerra (Kaldor, 1999).

8 Sandia National Laboratories, Los Alamos National Laboratory y el Lawrence Livermore National Laboratory.

9 El Lawrence Livermore National Laboratory, por ejemplo, redujo su investigación en armamento nuclear de la totalidad de sus actividades en los años cincuenta, a menos del $50 \%$ en el año 1997 (Tarter, 1997).

10 Datos Annuaire statistique de la Défense, 2002.

\section{BIBLIOGRAFÍA}

Adams, G. (1982): The Politics of Defense Contracting. The Iron Triangle, New Brunswick and London, Transaction Publishers.

Altman, J. (2006): Military Nanotechnology: Potential Applications and Preventive Arms Control, London/New York, Routledge.

CORDIS (2006): Verheugen makes the case for security research in FP7, Retrieved
5 mayo 2006, from http://cordis.europa.eu/fetch?CALLER=NEWS SECURITY ALL\&ACTION=D\&OM EN RCN $A=25244$.

Crease, R. P. (1993): "The National Labs and Their Future," Forum for Applied Research and Public Policy (Winter), 96-104.

Cuthbertson, I. (1983): Response of HMG to the questions posed in Working Paper 2 (Revision 2) of the UN Study on Military Research and Development, Arms Control and Disarmament Unit, Foreign and Commonwealth Office.

DeGrasse Jr., R. W. (1983): Military Expansion, Economic Decline. The Impact of Military Spending on U.S. Economic Performance, Armonk, New York, Sharpe.

DeLauer, R. (1984): "Technology Development and Transfer", Commercializing Defense Related Technology, R. L. Kuhn, New York, Praeger.

Edwards, P. N. (1997): The Closed World. Computers and the Politics of Discourse in Cold War America, Cambridge, Ma., and London, The MT Press.

Eisenhower, D. D. (1961): "Farewell address by President Dwight D. Eisenhower", Box 38, Speech Series, Papers of Dwight D. Eisenhower as President 1953-61, Eisenhower Library, National Archives and Records Administration.

Feldman, J. M. (1999): "Civilian diversification, leaming and insititutional change: growth through knowledge and power", Environment and Planning, 31 (10), 1805-1824.

Flamm, K. (1987): Targeting the Computer, Washington D.C., Brookings Institution.

Galbraith, J. K. (1985): The New Industrial State, Boston, Houghton Mifflin.

Gansler, J. S. (1995): Defense Conversion. Transforming the Arsenal of Democra$c y$, Cambridge, Ma. and London, MIT Press.
Hampson, F. (1989): Unguided missiles. How American buys its weapons, New York and London, W. W. Norton.

Kaldor, M. (1982): The Baroque Arsenal, London, Andre Deutsch.

Kaldor, M. (1999): New and Old Wars: Organized Violence in a Global Era, Standford, Standford University Press.

Lerner, J. (1992): "The Mobility of Corporate Scientists and Engineers between Civil and Defense Activities - Implications for Economic Competitiveness in the Post-Cold-War Era", Defence Economics, 3 (3), 229-242.

Leslie, S. W. (1993): The Cold War and American Science. The Military-IndustrialAcademic Complex at MIT and Standford, New York, Columbia University Press.

Manufacturing Studies Board (1987): Manufacturin Technology: Cornerstone of a Renewed Defense Industrial Base, Washington D.C., National Academy Press.

Melman, S. (1974): The Permanent War Economy. American Capitalism in Decline, New York, Simon and Schuster.

Mills, C. W. (1956): The Power Elite, New York, Oxford University Press.

Molas-Gallart, J. (2001): "Government policies and complex product systems: The case of defence standards and procurement", International Journal of Aerospace Management, 1 (3), 268-280.

Molas-Gallart, J. y Tang, P. (2006): "Ownership matters: intellectual property, privatization and innovation", Research Policy, 35 (2), 200-212.

O'Connell Jr., C. F. (1985): "The Corps of Engineers and the Rise of Modem Management, 1827-1856", Military Enterprise and Technological Change. Perspectives on the American Experience, M. R. Smith, Cambride, Ma. y London, MIT Press, 87-116.

O'Connell, R. L. (1989): Of Arms and Men. A History of War, Weapons, and Aggres- 
sion, New York, Oxford, Oxford University Press.

Peck, M. J. y Scherer, F. M. (1962): The Weapons Acquisition Process: An Economic Analysis, Boston, Ma., Graduate School of Business Administration, Harvard University.

Smith, M. R. (1987): "Army Ordenance and the 'American System' of Manufacturing, 1827-1856", Military Enterprise and Technological Change, Perspectives on the American Experience, M.
R. Smith, Cambridge, MA, MIT Press, 87-116.

Smith, M. R. (1987): "Introduction", Military Enterprise and Technological Change. Perspectives on the American Experience, M. R. Smith, Cambridge, MA, MIT Press, 1-37.

Stear, E. B. (1997): "Defense Conversion at Boeing", Defense Conversion Strategies, R. F. Dundervill, P. F. Gerity, A. K. Hyder y L. H. Luessen, Dordrecht, Kluwer Academic Publishers, 43-70.
Tarter, C. B. (1997): "Creating the Laboratory's Future. Director's Statement", Creating the Laboratory's Future. A Strategy for Lawrence Livermore National Laboratory, http://www.IInl. gov/director/ar128045/020director. htm.

U.S. Congress Office of Technology Assessment (1989): Holding the Edge: Maintainig the Defense Technology Base, U.S. Government Printing Office. 